\title{
Revisiting poromechanics in the context of microporous materials
}

\section{Poromécanique revisitée dans le contexte des matériaux microporeux}

\author{
Gilles Pijaudier-Cabot, Romain Vermorel ${ }^{1}$, Christelle Miqueu, and Bruno Mendiboure \\ Laboratoire des Fluides Complexes et leurs Réservoirs (UMR 5150), Université de Pau et des \\ Pays de l'Adour, Allée du Parc Montaury, F-64600 Anglet
}

Abstract:

Poromechanics offers a consistent theoretical framework for describing the mechanical response of porous solids fully or partially saturated with a fluid phase. When dealing with fully saturated microporous materials, which exhibit pores of the nanometer size, aside from the fluid pressure acting on the pore walls additional effects due to adsorption and confinement of the fluid molecules in the smallest pores must be accounted for. From the mechanical point of view, these phenomena result into volumetric deformations of the porous solid: the so-called "swelling" phenomenon. The present work investigates how the poromechanical theory should be refined in order to describe adsorption and confinement induced swelling in microporous solids. Firstly, we report molecular simulation results that show that the pressure and density of the fluid in the smallest pores are responsible for the volumetric deformation of the material. Secondly, poromechanics is revisited in the context of a microporous material with a continuous pore size distribution. Accounting for the thermodynamic equilibrium of the fluid phase in the overall pore space, the new formulation introduces an apparent porosity and an interaction free energy. We use a prototype constitutive relation relating these two quantities to the Gibbs adsorption isotherm, and then

\footnotetext{
${ }^{1}$ Corresponding author: romain.vermorel@univ-pau.fr
} 
calculate the induced deformation of the solid matrix. Agreement with experimental data found in the literature is observed. As an illustrating example, we show the predicted strains in the case of adsorption of methane on activated carbon.

Keywords:

Porous media; poromechanics; microporous materials; swelling; adsorption; fluid confinement; poroelasticity.

Résumé:

La poromécanique offre un formalisme théorique adapté à la description de la réponse mécanique des solides poreux, complètement ou partiellement saturés par une phase fluide. Dans le cas de matériaux microporeux comportant des pores de taille nanométrique, outre la pression du fluide s'exerçant sur les parois des pores, des effets additionnels dus à l'adsorption et au confinement du fluide dans les pores de plus petites tailles doivent être pris en compte. Du point de vue mécanique, ces phénomènes résultent en un gonflement du solide poreux. Ce travail s'intéresse à la manière dont le formalisme poromécanique doit être affiné dans le but de décrire les phénomènes de gonflement induits par l'adsorption et le confinement de fluide dans un matériau microporeux. Tout d'abord, nous rapportons des résultats de simulations moléculaires qui montrent que l'état de pression et de densité du fluide contenu dans les plus petits pores est responsable des déformations volumiques du milieu. Dans un deuxième temps, nous étendons le formalisme poromécanique au cas d'un matériau microporeux présentant une distribution continue de tailles de pores. En exprimant la condition d'équilibre thermodynamique du fluide dans tout le volume poreux, deux termes sont introduits dans le nouveau formalisme: la porosité apparente et l'énergie libre d'interaction. Nous proposons alors une relation constitutive simple, liant ces deux quantités à l'isotherme d'adsorption de Gibbs, et nous calculons les déformations résultantes du squelette 
poreux en bon accord avec les données expérimentales rencontrées dans la littérature. A titre d'exemple, nous montrons les déformations prévues dans le cas de l'adsorption de méthane dans des charbons actifs.

\section{Mots Clés:}

Milieux poreux; poromécanique; matériaux microporeux; gonflement; adsorption; confinement de fluide; poroélasticité.

\section{Introduction}

Poromechanics offers a consistent theoretical framework for describing the mechanical response of porous solids saturated, or partially saturated with a fluid phase. The theory is based upon the superposition of the solid and liquid phases. In the case of fully saturated porous solids, it is assumed that the fluid-solid interaction is restricted to the influence of the pressure on the inner surface of the porous material. In partially saturated porous solids, additional forces, i.e. capillary forces are introduced. Many authors have used this modern theoretical framework, which is thoroughly described e.g. in the textbooks by Coussy $[1,2]$. Microporous materials are, according to IUPAC standards, solids with pores of the nanometer size. Coal, activated carbons, zeolites, cement paste or tight rocks are among those materials which can be used in chemical engineering processes, in building construction, or may be encountered in the production of gas from very tight reservoirs or coal seams (Coal Bed Methane - CBM). Aside from the classical fluid-solid interaction observed in macroporous materials, there are additional effects that should be considered in the case of a material with very small pores filled with a fluid phase. Two features should be distinguished: adsorption and the fluid confinement: (i) adsorption becomes important because the inner surface of the pores is very large and surface forces cannot be neglected anymore; (ii) in very small pores, 
the molecules of fluid are confined. Interaction between molecules of fluid is modified, it cannot develop in the same way as if the fluid would be placed in a large container. This effect includes fluid-fluid and fluid-solid interactions.

From the mechanical point of view, these phenomena result into volumetric deformations of the porous solid. Swelling is commonly observed during sorption-desorption of several gases such as carbon dioxide or methane in charcoal, see e.g. the paper by Levine [3] although seminal experimental works of Meehan [4] and Bangham and Fakhroury [5] date back to the 1920 s.

For meso-porous materials (pores of size in the range of tenth of nanometers), Gor and Neimark [6] used Derjaguin theory of thin film equilibrium in order to devise a simple mechanical model describing the volumetric deformation of a porous solid during sorptiondesorption of nitrogen. Volumetric deformations were considered to be proportional to the socalled solvation pressure, the difference between the adsorption-induced stress and the external pressure in the bulk fluid.

$\mathrm{CO}_{2}$ swelling of coal and carbon absorbents has been recently analysed more thoroughly within the framework of poromechanics. Vandamme et al. [7] extended poromechanics to surface effects adding energy stored at the solid-fluid interface in the formulation. Although the model is laid down in a quite general perspective, the interaction stresses are later related to the Langmuir adsorption isotherm, which means that interactions are restricted to adsorption effects (mesopores). Molecular simulations are used for computing the adsorption isotherm for mesoporous and nanoporous materials. Results point out the influence of the nanopores on swelling but contradict, to some extend, the good agreement observed with the Langmuir isotherm where confinement effects are not accounted for. Mushrif and Rey [8] followed some similar reasoning as far as the poromechanics formulation is concerned. They computed the adsorption-induced strain directly from the chemical potential of the adsorbate, 
more specifically from the difference between the chemical potential of the fluid in the strained and unstrained absorbent. Good agreement with swelling data on activated carbon particles was observed.

In the above approaches, the inner pressure applied by the fluid on the solid phase was left somehow unspecified. Let us consider a porous saturated solid placed in a container filled with the fluid at a bulk pressure (bulk solution). The pressure inside the pores computed is different from the bulk pressure, as a result of adsorption and confinement [9]. It is very seldom that the two quantities are distinguished. Furthermore, the difference should depend on the bulk pressure, on the temperature, and on the pore size. In a solid with a continuous pore size distribution, inner pressures are different from one pore size to another, and different from the bulk pressure if the pores are sufficiently small.

The purpose of this note is twofold: firtsly, the effect of the adsorption and confinement on the pressure and mass density of a fluid filling nanopores is illustrated from existing molecular simulations results. We show that small pores have indeed a large effect on swelling. Secondly, poromechanics is revisited in the context of a microporous material with a continuous pore size distribution. The fluid inside the pores is assumed to be in chemical equilibrium, thus the chemical potential is used in the formulation, which refers to the bulk reference pressure of the fluid. A prototype, linear constitutive relation is discussed.

\section{Poromechanics with results from molecular simulations}

According to classical poromechanics, the free (Helmoltz) energy of a porous solid saturated with a fluid is written as the sum of the free energy of the solid phase $\psi_{s}$ and of the specific energy of the fluid phase $\psi_{f}$ :

$$
\psi=\psi_{s}+m_{f} \psi_{f}
$$


where $m_{f}$ is the mass of fluid inside the pores. The entropy of the system is split according to the same principle. The mechanical dissipation rate is computed using the state laws of the fluid. In the reversible case, we obtain the free energy of the solid phase:

$$
\frac{d \psi_{s}}{d t}=\sigma: \frac{d \varepsilon}{d t}+p \frac{d \varphi}{d t}-S_{s} \frac{d T}{d t}
$$

where $\sigma$ is the stress tensor, $\varepsilon$ is the strain tensor, $p$ is the fluid pressure, $\varphi$ is the porosity, $S_{s}$ is the entropy of the solid skeleton and $T$ is the temperature. We further restrict considerations to infinitesimal reversible transformations at constant temperature in an isotropic material. The free energy potential is a function of the strain and of the porosity. Classically, the constitutive equations read:

$$
\begin{aligned}
& \sigma_{i j}=\frac{\partial \psi_{s}}{\partial \varepsilon_{i j}}=\left[\left(K+b^{2} N\right) \varepsilon_{k k}-b N \varphi\right] \delta_{i j}+2 G e_{i j}, \text { with } \varepsilon_{i j}=e_{i j}+\frac{\varepsilon_{k k}}{3} \delta_{i j} \\
& p=\frac{\partial \psi_{s}}{\partial \varphi}=-b N \varepsilon_{k k}+N \varphi
\end{aligned}
$$

where $K$ is the modulus of incompressibility, $b$ is the Biot coefficient, and $N$ is the Biot modulus. $\delta_{i j}$ is the Kronecker symbol.

Consider now that the porous saturated solid is placed in a container filled with the saturating fluid at bulk pressure $p_{b}$. The hydrostatic stress applied by the fluid at bulk pressure on the solid is $\sigma_{i j}=-p_{b} \delta_{i j}$, but the pore pressure $p$ is different from the bulk pressure due to adsorption and confinement effects. Eqs. 3, in their incremental form yield the volumetric deformation increment denoted as $d \varepsilon$ as a function of the increments of pore and bulk pressures:

$$
d \varepsilon=d \varepsilon_{k k}=\frac{1}{K}\left(-d p_{b}+b d p\right)=\frac{d p_{b}}{K}\left(b \frac{\partial p}{\partial p_{b}}-1\right)
$$

The derivative of the pore pressure with respect to the bulk has been introduced in the formulation, as the pore pressure is not expected to depend on other parameters (at constant temperature as for a given pore size distribution). It is this term that is going to be obtained 
from molecular simulations. We use here the results from Mendiboure and Miqueu [9] (see also Ref. [10]) and consider a slit pore of width $2 R$ as shown in Fig. 1. Monte Carlo simulations are performed in the Grand Canonical ensemble. The fluid-fluid interactions are described with 12-6 Lennard-Jones potential and the solid-fluid interaction by the integrated 10-4-3 potential. The pore pressure $p$ is the component perpendicular to the surface of the pore. In confined fluids, the pressure is not a scalar and the tangent and normal pressure to the surface of the pore are different. The normal pressure $p$ is computed as described by Varnik et al. [11]. In the calculation, the solid phase is graphite and the fluid phase is methane at $353 \mathrm{~K}$. Figure 2 (a) shows the pore pressure versus the bulk pressure for several pore sizes. This is clearly a nonlinear relation which is very much dependent on the pore size $R$. For a bulk pressure equal to $2 \mathrm{MPa}$, the evolution of the pore pressure with the pore width is plotted in Fig. 2 (b). Oscillations corresponding to the structuration of the fluid into successive layers are observed. Note the range of variation of the pore pressure, with very high values for small pore sizes.

Substitution of these results into Eq. (4) yields the corresponding volumetric deformation of the solid phase. This substitution may be performed with and without step-by-step correction for the variation of porosity due to the skeleton deformation. Fig. 4 shows the results for a monodisperse porous material with pore radii equal to 0.348 and $1.252 \mathrm{~nm}$. The dotted curve is the calculation without step-by-step correction for the variation of porosity. We took $K=3$ $\mathrm{GPa}, b=0,95$ and the initial porosity $\varphi_{0}=0.88$, which corresponds to typical values for activated carbon. Due to the large variations of pore pressure as a function of the pore radius, the volumetric deformations may be very different. In the present case, experiments on charcoal exhibit deformations of the order of $0.1 \%$. This can only be achieved by taking into account contribution from very small pores (a few tenths of nanometers) (Fig. 3 (a)). For pores of the nanometer range (Fig. 3 (b)), the volumetric deformations are much less than the 
expected values. Of course, the present results are indicative and order of magnitude should be regarded only, but the same conclusion was also observed in Ref. [7] in which the adsorption isotherm rather than the pore pressure was derived from molecular simulations.

\section{Linear poromechanics of microporous solids}

Extending poromechanics to the case of microporous material means that adsorption and confinement effects ought to be included in the formulation. Thus, a difficulty is faced: in a solid with a continuous pore size distribution, the pore pressure is not unique and depends on the geometry of the pore. In each pore, however, the interstitial fluid is in equilibrium with the fluid contained in adjacent connected pores. This equilibrium condition means that the fluid in each pore is at the same chemical potential $\mu_{f}$. Same as in the previous section, we shall consider a saturated porous solid immersed in a fluid at a bulk pressure $p_{b}$ and mass density $\rho_{b}$. Equilibrium means:

$$
\mu_{f}=\psi_{f}+\frac{p}{\rho}=\mu_{f b}=\psi_{f b}+\frac{p_{b}}{\rho_{b}}
$$

where the chemical potential $\mu_{f}\left(\mu_{f b}\right)$ of the interstitial fluid (bulk solution) is expressed as a function of the specific fluid free energy $\psi_{f}\left(\psi_{f b}\right)$, pressure $p\left(p_{b}\right)$, and density $\rho\left(\rho_{b}\right)$ of the interstitial fluid (bulk solution).

\subsection{Free energy of the microporous saturated skeleton}

In the general expression of the mechanical dissipation $\phi_{s}$ (see the derivation in Ref. [1]), the chemical potential replaces the free specific enthalpy of the fluid:

$$
\frac{d \phi_{s}}{d t}=\sigma: \frac{d \varepsilon}{d t}+\mu_{f} \frac{d m_{f}}{d t}-S \frac{d T}{d t}-\frac{d \psi}{d t}
$$

where $S$ is the total entropy (solid and fluid), $m_{f}$ is the mass of fluid in the system and $\psi$ is the total free energy. Substitution of Eqs. $(1,5)$ in Eq. (6) and restriction to isothermal transformations yields: 


$$
\frac{d \phi_{s}}{d t}=\sigma: \frac{d \varepsilon}{d t}+\left(\psi_{f b}+\frac{p_{b}}{\rho_{b}}\right) \frac{d m_{f}}{d t}-\frac{d \psi_{s}}{d t}-\frac{d\left(m_{f} \psi_{f}\right)}{d t}
$$

It is further possible to express the free energy of the fluid inside each pore as a function of quantities related to the bulk phase. For this, we use again Eq. (5):

$$
\psi_{f}=\psi_{f b}+\frac{p_{b}}{\rho_{b}}-\frac{p}{\rho}
$$

This equation is valid for each pore size. In order to obtain the total specific free energy of the fluid, we need to perform the summation over the lagrangian material volume $\Omega_{0}$ :

$$
m_{f} \psi_{f}=\frac{1}{\Omega_{0}} \int_{\Omega_{0}}\left[\psi_{f b}+\frac{p_{b}}{\rho_{b}}-\frac{p(R)}{\rho(R)}\right] \rho(R) \varphi(R) d \Omega_{0}
$$

We have introduced in the above expression $\varphi(R)$, the relative volume of pores of size $R$ (volume divided by the total volume of pores). Eq. (9) may be further simplified:

$$
\begin{aligned}
& m_{f} \psi_{f}=\left[\psi_{f b}+\frac{p_{b}}{\rho_{b}}\right] \int_{\Omega 0} \rho(R) \varphi(R) \frac{d \Omega_{0}}{\Omega_{0}}-\int_{\Omega 0} p(R) \varphi(R) \frac{d \Omega_{0}}{\Omega_{0}} \\
& =m_{f} \psi_{f b}+\left[m_{f} \frac{p_{b}}{\rho_{b}}-\int_{\Omega 0} p(R) \varphi(R) \frac{d \Omega_{0}}{\Omega_{0}}\right]=m_{f} \psi_{f b}+\psi_{\text {int }}
\end{aligned}
$$

where $\psi_{\text {int }}$ is the term in the free energy resulting from adsorption and confinement. We may now substitute this equation in the expression of the dissipation in the solid skeleton Eq. (7). We use the state laws of the fluid in the bulk phase and obtain the expression:

$$
\frac{d \phi_{s}}{d t}=\sigma: \frac{d \varepsilon}{d t}+p_{b} \frac{d\left(\int_{\Omega 0} \rho(R) \frac{\varphi(R)}{\rho_{b}} \frac{d \Omega_{0}}{\Omega_{0}}\right)}{d t}-\frac{d \psi_{s}}{d t}-\frac{d\left(\psi_{\mathrm{int}}\right)}{d t}
$$

We may now define the apparent porosity $\varphi^{*}$ of the microporous material:

$$
\varphi^{*}=\int_{\Omega 0} \rho(R) \frac{\varphi(R)}{\rho_{b}} \frac{d \Omega_{0}}{\Omega_{0}}
$$

and in the case of reversible transformation, the free energy of the skeleton reads:

$$
\frac{d \psi_{s}}{d t}=\sigma: \frac{d \varepsilon}{d t}+p_{b} \frac{d\left(\varphi^{*}\right)}{d t}-\frac{d\left(\psi_{\text {int }}\right)}{d t}
$$


This expression differs from the standard expression in porous materials (Eq. 2). The bulk pressure of the saturating fluid appears, but the porosity is modified in order to account for the effect of confinement and adsorption on mass density. Same as in Ref. 7, a interaction energy is introduced. In the present case, we have related this energy to the "true" pore pressure distribution (Eq. 10).

\subsection{Simplified model}

In order to proceed, the interaction term in the free energy of the skeleton needs to be further investigated, e.g. by relating the internal pore pressure to the state variables (strain, apparent porosity...) or using results from molecular simulations. We shall leave this issue for further studies and switch to a more phenomenological simplified modeling. The interaction energy is set as a function of the apparent porosity of the material only.

$$
d\left(\psi_{\text {int }}\right)=p_{\text {int }} d \varphi^{*} \text { where } p_{\text {int }}=\frac{\partial \psi_{\text {int }}}{\partial \varphi^{*}}
$$

where $p_{\text {int }}$ is an interaction pressure. The deformation of the skeleton is considered to have a negligible influence. Assuming that the free energy of the skeleton is now a function of the strain and of the apparent porosity, we may derive the state laws following the same steps as in standard poromechanics. We obtain in the reversible regime ( $p_{\text {int }}$ is a linear function of $\left.\varphi^{*}\right)$ :

$$
\begin{aligned}
& \sigma_{i j}=\frac{\partial \psi_{s}}{\partial \varepsilon_{i j}}=\left[\left(K+b^{2} N\right) \varepsilon_{k k}-b N \varphi^{*}\right] \delta_{i j}+2 G e_{i j}, \text { with } \varepsilon_{i j}=e_{i j}+\frac{\varepsilon_{k k}}{3} \delta_{i j} \\
& p_{b}-p_{\text {int }}=\frac{\partial \psi_{s}}{\partial \varphi^{*}}=-b N \varepsilon_{k k}+N \varphi^{*}
\end{aligned}
$$

Note that the coefficients entering in the constitutive relations are not necessarily the same as those in section 2. More specifically, they must depend on the bulk pressure if $p_{\text {int }}$ is not a linear function of $\varphi^{*}$. We may also compute the volumetric deformation resulting from the immersion of the microporous solid in the saturation fluid at bulk pressure: 


$$
d \varepsilon=\frac{1}{K}(b-1) d p_{b}-\frac{d p_{\text {int }}}{K}
$$

We need now to compute the interaction pressure. We shall relate this quantity to the Gibbs excess isotherm as observed experimentally in early papers [4,5]. By definition, the Gibbs excess isotherm $\Gamma\left(p_{b}, T\right)$ is providing the difference between the quantity of fluid molecules inside the porous material and the quantity of fluid molecules occupying the same volume at the bulk pressure:

$$
\begin{aligned}
& \Gamma\left(p_{b}, T\right)=\frac{1}{M \rho_{a p p}}\left(\int_{\Omega 0} \rho(R) \varphi(R) \frac{d \Omega_{0}}{\Omega_{0}}-\int_{\Omega 0} \rho_{b} \varphi(R) \frac{d \Omega_{0}}{\Omega_{0}}\right) \\
& =\frac{1}{M} \frac{\rho_{b}}{\rho_{a p p}}\left(\varphi^{*}-\int_{\Omega 0} \varphi(R) \frac{d \Omega_{0}}{\Omega_{0}}\right)=\frac{1}{M} \frac{\rho_{b}}{\rho_{a p p}}\left(\varphi^{*}-\varphi\right)
\end{aligned}
$$

where $M$ is the molar mass of the adsorbed gas and $\rho_{a p p}$ the apparent density of the porous material. This excess results from adsorption and confinement effects. If the excess is zero, it means that adsorption and confinement effects are negligible. Consequently, the interaction energy and interation pressure should also vanish and the standard poromechanical formulation should be recovered. Thus, we shall assume in the present simplified approach that the interaction pressure is proportional to the Gibbs excess isotherm:

$$
p_{\mathrm{int}}=-k \Gamma\left(p_{b}, T\right)=-\frac{k}{M} \frac{\rho_{b}}{\rho_{a p p}}\left(\varphi^{*}-\varphi\right)
$$

where $k$ is a model parameter. It is not necessarily a constant (and might depend on the bulk pressure for instance) and it should be fitted from swelling test data.

We consider first the experiments performed by Levine on the adsorption of supercritical methane on charcoal [3]. We use the measured Gibbs excess isotherm shown in Fig. 4 (a) to calculate the strain from Eq. (16) and Eq. (18). Comparison between the calculated strain and experimental data is shown in Fig. 4 (b). We took $K=3 \mathrm{GPa}, b=0,95, \rho_{\text {app }}=500 \mathrm{~kg} \cdot \mathrm{m}^{-3}$, and $M=16.043$ g.mol ${ }^{-1}$ corresponding to microporous coal. The model parameter $\mathrm{k}$ was set constant, $k=13.8 \mathrm{GPa} . \mathrm{g} \cdot \mathrm{mol}^{-1}$. The theoretical predictions of swelling are in good agreement 
with experimental data (Fig. 4b). In Eq. (16) we can reformulate the coefficient of the first right hand member as $(1-b)=K / K_{s}$ (see Ref. [1]), where $K_{s}$ is the incompressibility modulus of the coal solid matrix itself. For microporous coal, the order of magnitude of $K_{s}$ is $90 \mathrm{GPa}$ [7], which results in $K / K_{s}<<1$. This means that the contribution of the bulk pressure in Eq. (16) can be neglected, and thus the incremental strain is almost proportional to the increment of interaction pressure $p_{\text {int }}$. The proportionality between the volumetric deformation and the adsorption isotherm was observed experimentally by Levine [3]. Bangham and Fakhroury [5] argued that a square root relationship between the adsorbed quantity and the volumetric deformation provided better results but they considered chemical sorption of water in charcoal. In the case of physi-sorption, the proportionality set in Eq. (18) is a crude, yet credible, simplifying assumption.

As a second illustrating example, let us now consider the case of an activated carbon (Ecosorb). Typical Gibbs excess isotherms are shown in Fig. 5 (a). They have been measured with a manometric technique depicted in Ref. [12]. Figure 5 (b) shows the volumetric strain obtained from Eq. (16) and (18) with $k=13.8$ GPa.g.mol ${ }^{-1}$. The volumetric deformation falls in the range of experimental results on activated carbon [8] and charcoal [3]. Of course, more accurate fits could be obtained by considering that this parameter depends on the solid skeleton properties, on the skeleton PSD, and on the nature of the adsorbed gas. In the present simplified model, orders of magnitude are targeted, prior to more detailed future investigations of the interaction energy.

\section{Summary and conclusions}

(1) Adsorption and confinement effects in microporous materials induce a variation of the pore pressure acting inside the solid skeleton. This pore pressure may reach very high values and it is an oscillating function of the pore size. The introduction of such a pore 
pressure in standard poromechanics shows the important influence of very small pores on the swelling of such materials, measured when the skeleton is saturated with the fluid.

(2) In order to account for adsorption and confinement effects, the thermodynamics based formulation of the porous solid saturated with the fluid needs to be modified. In each pore, independently of its size, the fluid is in equilibrium with that contained into connected adjacent pores. Their chemical potentials are equals and the free energy of the interstitial fluid is rewritten accordingly:

$$
m_{f} \psi_{f}=m_{f} \psi_{f b}+\psi_{\mathrm{int}}
$$

where a interaction energy $\psi_{\text {int }}$ appears, which results from adsorption and confinement effects in the micropores. Accounting for the continuous pore size distribution $\varphi(R)$ of the microporous material, the interaction energy is related to the true pore pressure distribution $p(R)$ as follows

$$
\psi_{\mathrm{int}}=m_{f} \frac{p_{b}}{\rho_{b}}-\int_{\Omega 0} p(R) \varphi(R) \frac{d \Omega_{0}}{\Omega_{0}}
$$

By substituting the expression of the fluid free energy in the skeleton dissipation, and considering isothermal reversible transformations, the free energy of the skeleton writes as

$$
\frac{d \psi_{s}}{d t}=\sigma: \frac{d \varepsilon}{d t}+p_{b} \frac{d\left(\varphi^{*}\right)}{d t}-\frac{d\left(\psi_{\mathrm{int}}\right)}{d t}
$$

where the corrected porosity $\varphi^{*}$, which takes account for fluid confinement effects is defined as

$$
\varphi^{*}=\int_{\Omega 0} \rho(R) \frac{\varphi(R)}{\rho_{b}} \frac{d \Omega_{0}}{\Omega_{0}} .
$$


(3) In a simplified model, the interaction energy is a function of the corrected porosity only. An interaction pressure derives from the interaction energy, considered as a potential through the following relation:

$$
p_{\text {int }}=\frac{\partial \psi_{\text {int }}}{\partial \varphi^{*}}
$$

As a result, the incremental volumetric strain depends on the interaction pressure as follows

$$
d \varepsilon=\frac{1}{K}(b-1) d p_{b}-\frac{d p_{\text {int }}}{K} .
$$

The interaction pressure is further related to the Gibbs excess isotherm data through the following empirical linear constitutive relation:

$$
p_{\text {int }}=-k \Gamma\left(p_{b}, T\right)
$$

Comparison of the model predictions with swelling test data from the literature exhibits the consistency of this approach. Illustration on an activated carbon shows that the model may provide the correct order of magnitude of the volumetric deformation as a function of the fluid bulk pressure.

Acknowledgements: Financial supports from ERC advanced Grant project Failflow (Ad-G 27769) and from Total SA under the Tight Gas project are gratefully acknowledged.

\section{References}

[1] O. Coussy, Poromechanics, John Wiley \& Sons, Chichester, 2004.

[2] O. Coussy, Mechanics and Physics of Porous Solids, John Wiley \& Sons, Chichester, 2010.

[3] J.R. Levine, Model study of the influence of matrix shrinkage on absolute permeability of coal bed reservoirs, J. Geol. Soc., Special publications, 109 (1996) 197-212. 
[4] F.T. Meehan, The expansion of charcoal on sorption of carbon dioxide, Proc. R. Soc. A, 115 (1927) 199-207.

[5] D.H. Bangham, N. Fakhroury, The expansion of charcoal accompagnying sorption of gases and vapours, Nature, 122 (1928) 681-682.

[6] G.Y. Gor, A.V. Neimark, Adsorption-induced deformation of mesoporous solids, Langmuir, 26 (16) (2010) 13021-13027.

[7] M. Vandamme, L. Brochard, B. Lecampion and O. Coussy, Adsorption and strain : The CO2 induced swelling of coal, J. Mech. Phys. Solids, 58 (2010) 1489-1505.

[8] S.H. Mushrif, A.D. Rey, An integrated model for adsorption-induced strain in microporous solids, Chem. Eng. Sci., 64 (2009) 4744-4753.

[9] B. Mendiboure, C. Miqueu, Evaluation of the pressure tensor of fluids confined into slit micropores, J. Chem. Phys., submitted (2011).

[10] A. Knorst-Fouran, Contribution à l'étude de propriétés interfaciales d'alcanes confinés par simulation moléculaire de type Monte Carlo, Ph.D. Université de Pau et des Pays de l'Adour (2010).

[11] F. Varnik, J. Baschnagel, K. Binder, Molecular dynamics results on the pressure tensor of polymer films, J. Chem. Phys., 113 (10) (2000) 4444-4453.

[12] A. Mouahid, D. Bessières, F. Plantier, G. Pijaudier-Cabot, A thermostated coupled apparatus for the simultaneous determination of adsorption isotherms and differential enthalpies of adsorption at high pressure and high temperature, J. Therm. Anal. Calorim., accepted. (DOI :10.1007/s10973-011-1820-2), (2011). 


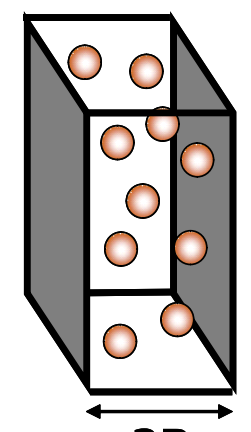

2R

Fig. 1: Model of graphite parallel slit pore used in GCMC simulations.

Fig. 1: Modèle de pore plan de graphite utilisé dans les simulations GCMC.
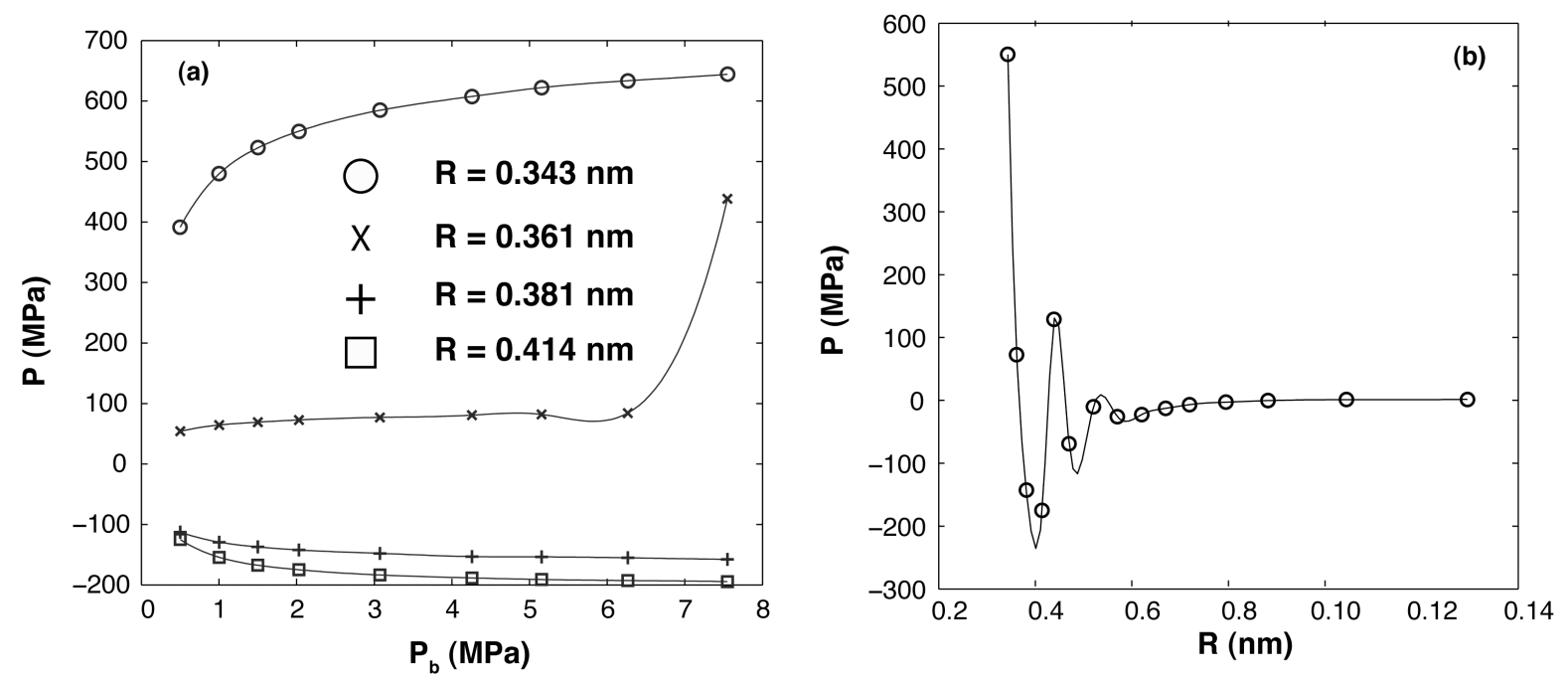

Fig. 2: (a) Component of the pore pressure perpendicular to the pore wall versus bulk pressure, for different pore sizes (methane into graphitic slit pores at 353K); (b) Component of the pore pressure perpendicular to the pore wall versus the pore size, for a bulk pressure equal to $2 \mathrm{MPa}$ (methane into graphitic slit pores at 353K). Dots stand for the molecular simulations results; solid lines stand for the interpolated data.

Fig. 2: (a) Pression perpendiculaire à la paroi du pore en fonction de la pression bulk, pour différentes tailles de pores (méthane dans des pores plans en graphite à 353K); (b) Pression perpendiculaire à la paroi du pore en fonction de la taille des pores, pour une pression bulk 
égale à $2 \mathrm{MPa}$ (méthane dans des pores plans en graphite à $353 \mathrm{~K})$. Les points représentent les résultats des simulations moléculaires; les lignes en trait plein montrent les données interpolées.
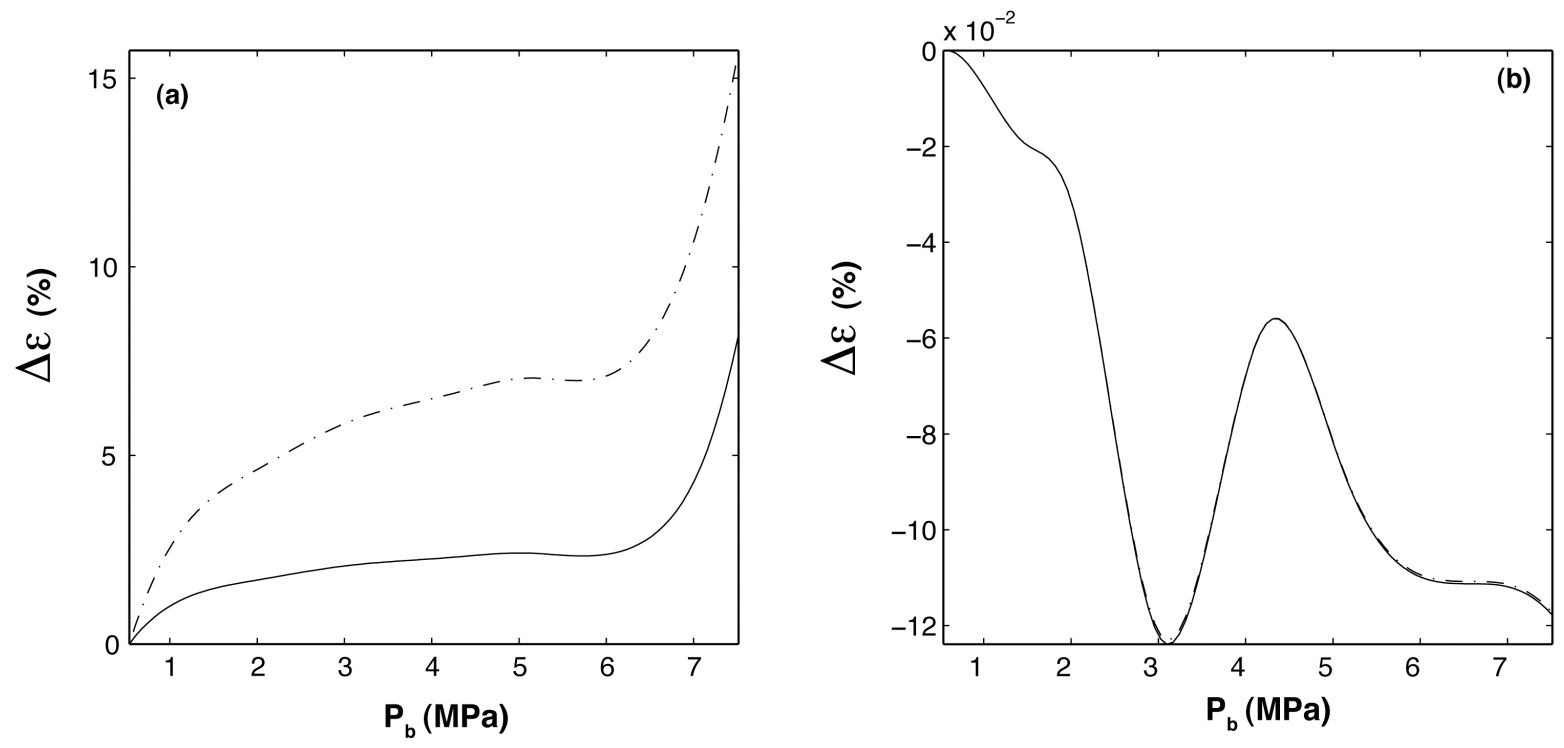

Fig. 3: Volumetric deformation of a monodisperse porous graphite containing methane at 353 K. Pore radii equal to $0.348 \mathrm{~nm}$ (a) and $1.252 \mathrm{~nm}$ (b). Dashed lines stand for the direct strain calculus; solid lines stand for the strain calculus with step-by-step correction of the porosity.

Fig. 3: Déformation volumique de graphite monodisperse contenant du méthane à $353 \mathrm{~K}$. Les rayons de pores représentés sont $0.348 \mathrm{~nm}$ (a) et $1.252 \mathrm{~nm}$ (b). Les lignes en trait mixte représentent le calcul de déformation direct; Les lignes en trait plein représentent le calcul de déformation avec correction pas à pas de la porosité. 

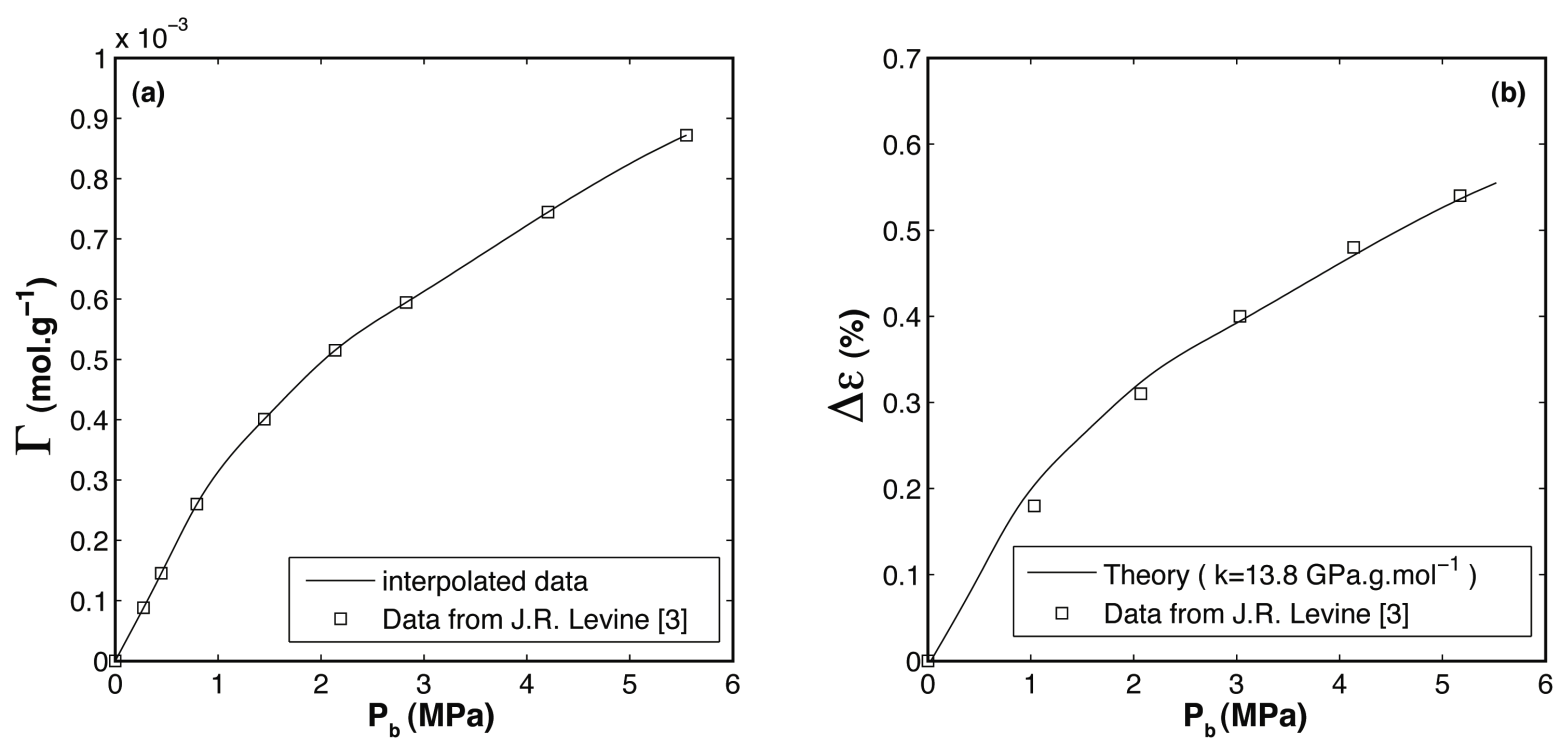

Fig. 4: (a) $\mathrm{CH}_{4}$ adsorption isotherm on Illinois charcoal (after [3]). (b) Evolution of the volumetric strain with the bulk pressure. Comparison between experimental data from Ref. [3] and the theoretical model prediction with the adjustable parameter $k=13.8 \mathrm{GPa} \cdot \mathrm{g} \cdot \mathrm{mol}^{-1}$.

Fig. 4: (a) Isotherme d'adsorption de $\mathrm{CH}_{4}$ dans du charbon d'Illinois (d'après [3]). (b)

Déformation volumique en fonction de la pression bulk. Comparaison entre les données expérimentales de Ref. [3] et les prédictions du modèle théorique avec le paramètre ajustable

$$
k=13.8 \mathrm{GPa} \cdot \mathrm{g} \cdot \mathrm{mol}^{-1} .
$$



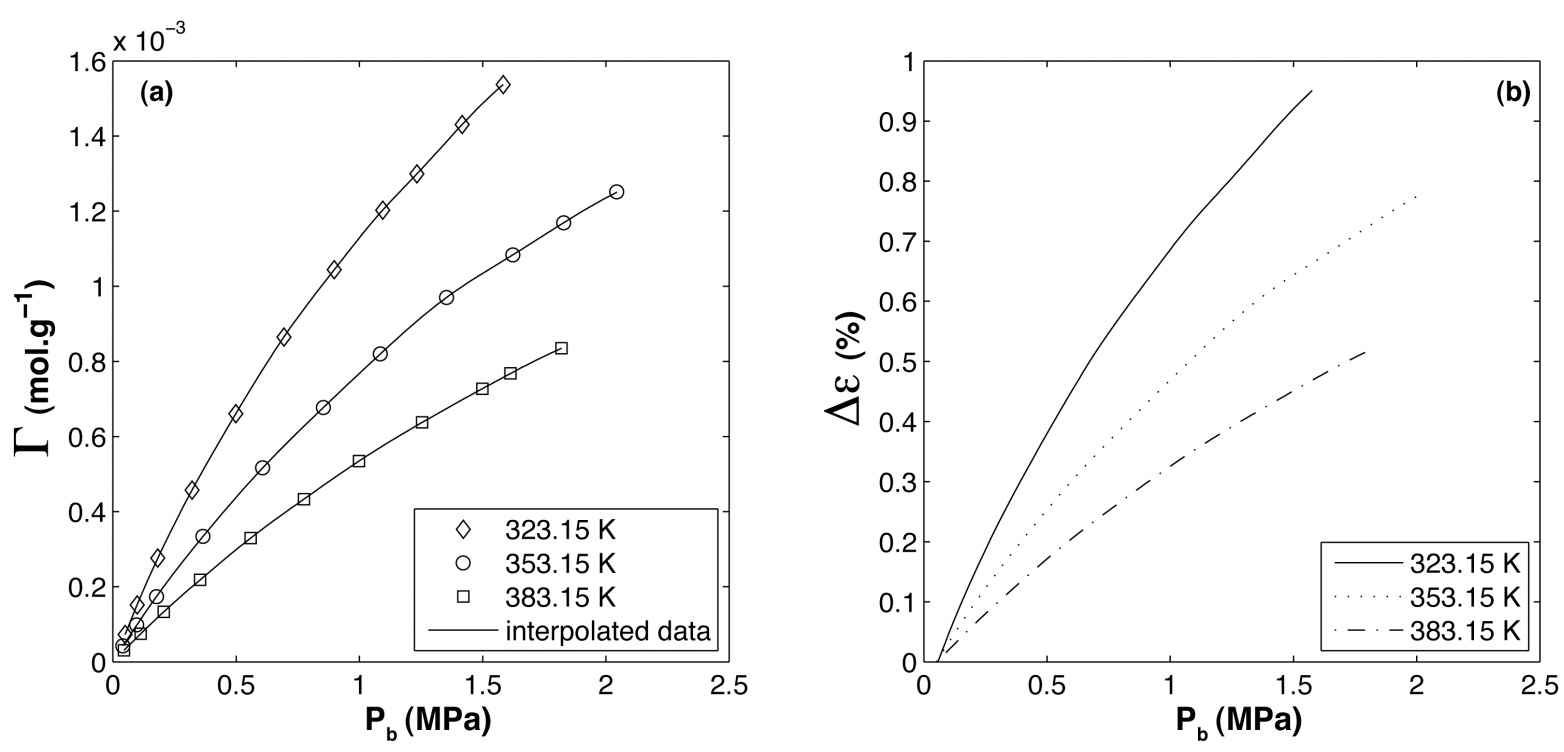

Fig.5: (a) $\mathrm{CH}_{4}$ adsorption isotherms on Ecosorb activated carbon. ( $\left.\diamond\right) 323.15 \mathrm{~K}$; (○) 353.15

$\mathrm{K}$; () $383.15 \mathrm{~K}$ (after [10]). (b) Volumetric strain obtained from adsorption isotherms, with the adjustable parameter $k=13.8 \mathrm{GPa} \cdot \mathrm{g} \cdot \mathrm{mol}^{-1}$.

Fig.5: (a) Isothermes d'adsorption de $\mathrm{CH}_{4}$ dans du charbon actif Ecosorb. ( $\left.\diamond\right) 323.15 \mathrm{~K}$; (○)

$353.15 \mathrm{~K}$; (口) $383.15 \mathrm{~K}$ (d'après [10]). (b) Déformation volumique obtenue à partir des isothermes d'adsorption, avec le paramètre ajustable $k=13.8 \mathrm{GPa} \cdot \mathrm{g} \cdot \mathrm{mol}^{-1}$. 